\title{
Exposición a plaguicidas y prácticas de uso y protección en embarazadas de zona rurales en control de atención primaria en la región de Valparaíso, Chile
}

\author{
Anamaría Silva D. 1,a, Mariana Arancibia H.2,b, Claudio Pulgar G. 3,c, Leyla Astorga

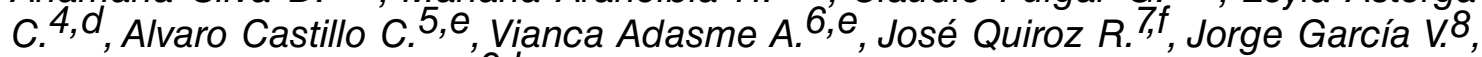 \\ María Fernanda Cavieres F.' ${ }^{9} h$
}

${ }^{1}$ Escuela de Obstetricia y Puericultura, Universidad de Valparaíso, Campus San Felipe. ${ }^{2}$ Escuela de Obstetricia y Puericultura, Universidad de Valparaíso. ${ }^{3}$ Departamento de Salud Municipalidad de Petorca, CESFAM Chincolco, Petorca. ${ }^{4}$ Departamento de Salud, Municipalidad de Quillota. ${ }^{5}$ CESFAM San Pedro, Quillota. ${ }^{6}$ CESFAM Chincolco, Petorca. ${ }^{7}$ CESFAM Chincolco, Petorca. ${ }^{8}$ Escuelas de Fonoaudiología y Obstetricia y Puericultura, Universidad de Valparaíso, Campus San Felipe. ${ }^{9}$ Escuela de Química y Farmacia, Universidad de Valparaíso. Chile.

a Socióloga, PhD. ${ }^{b}$ Matrona, MSc. ${ }^{c}$ Psicólogo, MSc. ${ }^{d}$ Asistente Social, MSc. ${ }^{e}$ Asistente Social. ${ }^{f}$ Tecnólogo Médico. $\mathrm{g}$ Estadístico. ${ }^{\mathrm{h}}$ Químico Farmacéutico, Toxicólogo, PhD.

Fuente de apoyo financiero: Fondo Nacional de Investigación y Desarrollo en Salud (FONIS; CONICYT-MINSAL). Proyecto SA12I2190.

\section{RESUMEN}

Antecedentes: Los trabajadores agrícolas y habitantes rurales están expuestos a productos plaguicidas. Aun cuando las políticas sanitarias se preocupan de normarlos, diversos estudios demuestran exposición en trabajadores y residentes cercanos a las zonas productivas. Entre ellos se encuentran mujeres gestantes, consideradas población particularmente vulnerable frente a plaguicidas. Objetivo: Identificar el contacto con plaguicidas, así como conductas asociadas a su uso, en mujeres gestantes de dos comunas agrícolas de la V Región en control prenatal en Centros de Salud Familiar. Método: Estudio observacional transversal, realizado en una muestra de ochenta gestantes que ingresaron a control entre mayo 2013 y abril 2014. El instrumento de recolección de datos fue una encuesta, aplicada por el equipo de salud al ingreso. Resultados: Las gestantes, en su gran mayoría, alguna vez han tenido contacto con plaguicidas, y cerca de la mitad lo tuvo en el mismo año en que se aplicó la encuesta. La exposición a plaguicidas se da tanto en los espacios productivos, como residenciales. Se observó que las medidas preventivas asociadas al contacto con agrotóxicos son insuficientes. A la vez, destaca la exposición a fumigaciones aéreas, frente a las cuales la posibilidad de prevención es aún menor. Conclusión: En comunas rurales puede existir un contacto permanente de la población femenina gestante con plaguicidas, lo cual sucede sin las medidas de protección adecuadas, incrementando el riesgo de exposición a estas sustancias. Es pertinente entonces, desarrollar una labor educativa activa para mejorar las prácticas de uso de plaguicidas.

\section{PALABRAS CLAVE: Plaguicidas, exposición materna, gestación, atención primaria de salud}

\section{SUMMARY}

Background: Agriculture workers and rural dwellers are exposed to pesticide products. Sanitation policies attempt to regulate their use, but studies demonstrate that a significant percentage of workers and residents who are close to the productive zones are exposed to these chemicals. Pregnant women are particularly 
vulnerable to pesticides. Aim: To identify the risk of exposure to pesticides and practices of pesticide handling in women under pregnancy control programs in Family Health Care Centers in two rural districts of the 5th Region. Method: Cross-sectional study, conducted during May 2013 and April 2014 on eighty women, who entered pregnancy control programs in either rural district. The health professionals of the Centers collected the data in a survey. Results: Most of the pregnant women declared having been in contact with pesticides at some point. Almost half of them had this contact in the same year of their interview, that is previous or during their pregnancy. The exposure to pesticides takes place in productive locations as well as residential areas. The preventive measures associated to the contact with pesticides are not sufficient. Rural populations are also exposed to aerial fumigations, which further limits preventive measures. Conclusion: Pregnant women living in a rural district may be permanently exposed to pesticides. Exposure to agrochemicals is not associated to safe prevention practices which increase the risk of exposure. Educational programs may aid to enhance safe pesticide use.

\section{KEY WORDS: Pesticides, maternal exposure, pregnancy, primary health care}

\section{INTRODUCCIÓN}

En Chile, existe notificación obligatoria de intoxicaciones agudas por plaguicidas (1), pero no existe ningún tipo de registro o monitoreo de intoxicación crónica. La cercanía entre zonas de actividad agrícola y zonas residenciales generan exposición no sólo ocupacional sino que también residencial, generando un riesgo especial en poblaciones vulnerables, tal como las gestantes y sus hijos e hijas por nacer. Un estudio cualitativo realizado en Quillota, Putaendo y Petorca (5 ${ }^{\mathrm{a}}$ Región, Chile) entre los años 2011-2012, reportó que ocurre exposición a plaguicidas de pequeños productores agrícolas y de la población residente porque no se toman suficientes medidas preventivas, a pesar de que existe conocimiento de que los plaguicidas pueden generar riesgos a la salud (2). En ese estudio también se evidenció temor a la fumigación aérea y una subestimación del riesgo de la fumigación terrestre.

La mayor parte de la evidencia sobre exposición a plaguicidas surge de estudios realizados en hombres, generando un vacío en el conocimiento necesario para evaluar el riesgo de exposición residencial y/o laboral en mujeres, más aún si se considera una posible influencia hormonal sobre los efectos inducidos por plaguicidas (3). Algunos estudios recientes llaman la atención sobre la falta de estudios que determinen la exposición a plaguicidas en poblaciones de riesgo como las mujeres embarazadas $(3,4,5)$, situación que también se repite en Chile.

El objetivo de esta investigación es identificar el contacto con plaguicidas así como las conductas asociadas a su uso y la prevención en embarazadas de dos comunas agrícolas de la $\mathrm{V}$ Región en control prenatal en Centros de Salud Familiar (CESFAM).

\section{SUJETOS Y MÉTODO}

El enfoque de este trabajo fue cuantitativo, de alcance descriptivo. La muestra consistió en 80 mujeres gestantes mayores de 18 años que ingresaron a control en los centro de Salud Familiar (CESFAM) de San Pedro (Quillota) y Chincolco (Petorca) pertenecientes a la $5^{\mathrm{a}}$ Región de Chile, y que aceptaron participar en el estudio, entre mayo de 2013 y abril de 2014. Esta muestra representa alrededor de un sesenta por ciento de la población anual atendida en los respectivos CESFAM.

Los datos fueron recolectados a través de una encuesta cerrada, basada en dimensiones levantadas en un estudio cualitativo previo (2) y que consideraba los indicadores de un formulario de diagnóstico y fiscalización, utilizado por la comisión conjunta del Servicio Agrícola Ganadero, Ministerio de Trabajo y Ministerio de Salud. El contenido de la encuesta fue revisado y testeado por profesionales expertos, incluyendo agrónomos y un estadístico, y fue piloteada para observación en términos de lenguaje, significados locales y duración. La encuesta validada y aplicada durante el estudio incluía las dimensiones siguientes: i. Antecedentes sociodemográficos, ii. Antecedentes gineco-obstétricos, iii. Contacto de las gestantes con plaguicidas, iv. Conductas de uso de plaguicidas (previo, durante y post aplicación de plaguicidas), y v. Molestias de salud derivadas del contacto con plaguicidas y resultado perinatal.

Este estudio es parte del proyecto FONISSA12I2190 "Impacto de la inclusión de un biomarcador para el monitoreo de plaguicidas organofosforados en el examen médico preventivo del adulto" (EMPA), y en el control gestacional, en población de zonas agrícolas de dos comunas de la 5ª Región, el cual fue aprobado por el Comité de Bioética de la Facultad de Medicina de la Universidad de Valparaíso. En términos de procedimiento, la encuesta, anónima, fue aplicada tras consentimiento informado a las gestantes por parte de las matronas en Petorca, y por el asistente social en Quillota. Las encuestadas eran libres de omitir alguna pregunta si así lo deseaban, por lo que el número total en algunas dimensiones encuestadas es menor que el número total del estudio. Los datos fueron ingresados en una base 
de datos y procesados mediante estadística descriptiva de tendencia central.

\section{RESULTADOS}

Antecedentes sociodemográficos de las gestantes. El total de las mujeres encuestadas se distribuyó de manera homogénea entre gestantes de la comuna de Petorca $(48,7 \%)$ y Quillota $(51,3 \%)$, cerca de dos tercios de las mujeres son menores de 30 años (64,9\%) y sólo un 12,6\% mayores de 35 años y por tanto con mayor riesgo obstétrico. La mayoría de las gestantes $(65,4 \%)$ tenían un nivel de formación medio con educación media o formación técnica completa y sólo un $15,4 \%$ con educación superior. Más de la mitad $(59,5 \%)$ al momento de la encuesta no era activa económicamente (por ser dueñas de casa, estudiantes o cesantes), o sólo tenían trabajo esporádico. Un $88,2 \%$ de las mujeres ocupadas eran trabajadoras, obreras o empleadas, ocupándose principalmente en el sector terciario (comercio y servicios, entre otros). Tan sólo 2,6\% de las mujeres eran empleadoras o patronas.

Antecedentes gineco-obstétricos. Las mujeres entrevistadas ingresaron tempranamente a control gestacional $(69,6 \%$ en el primer trimestre de gestación). Sólo 8,7\% se encontraban en el tercer trimestre. Algo más de un tercio (36,3\%) eran primíparas, mientras que un $56,2 \%$ habían tenido entre uno y dos partos previos a este embarazo. En cuanto a los antecedentes de otros hijos: un 10,3\% habían tenido hijos prematuros y un 5,3\% con malformaciones congénitas. Un 15,0\% había tenido abortos y un $8,0 \%$ hijos mortinatos.

Contacto de las gestantes con plaguicidas. En la Tabla I se indica el contacto directo con plaguicidas según lugar, momento y medios de aplicación. La gran mayoría de las mujeres señaló alguna vez haber vivido, permanecido o trabajado en un lugar donde se aplicaban plaguicidas, dándose el contacto reciente (este año) en un $41,9 \%$. No obstante, solo una minoría (12 mujeres; 9,6\%) declaró haber aplicado plaguicidas ella misma. Los lugares de contacto con plaguicidas más frecuentes fueron la empresa agrícola o invernadero. Otros lugares son el predio o invernadero de pequeña escala, o bien en el sector donde reside. Cuando habían estado en contacto con plaguicidas, la forma de aplicación más reportada fue la terrestre. Aproximadamente, una cuarta parte $(24,6 \%)$ señaló la fumigación aérea. En cuanto al momento del contacto directo, algo menos de la mitad lo ha tenido en el transcurso del año en que fue encuestada. Por otro lado, cerca de la mitad de las encuestadas tenían contacto indirecto con plaguicidas, por vivir con alguien que trabaja en un lugar donde se aplican plaguicidas, o porque se guardan plaguicidas en su lugar de residencia (Tabla II).

\section{Tabla I \\ CONTACTO DIRECTO CON PLAGUICIDAS, SEGÚN LUGAR, MOMENTO Y MEDIOS DE APLICACIÓN}

\begin{tabular}{cc}
\hline Contacto & $\begin{array}{c}\text { Porcentaje que ha } \\
\text { tenido contacto }\end{array}$ \\
\hline
\end{tabular}

Ha tenido contacto:

Sí $\quad 86,8$

No $\quad 13,2$

Total 100

$\mathrm{n}=76$

Lugar de contacto*:

Predio y/o invernadero no em- 32

presarial

Empresa agrícola/invernadero $\quad 66,1$

de empresa

Sector donde reside $\quad 33,8$

Otro 3,3

$\mathrm{n}=65$

Momento del contacto*:

Este año $\quad 41,9$

El año pasado $\quad 33,9$

Hace más de dos años $\quad 62,9$

$\mathrm{n}=62$

Medios de aplicación*:

Bomba manual o de espalda $\quad 50,8$

Con tractor $\quad 52,5$

Fumigación aérea $\quad 24,6$

$\mathrm{n}=61$

* Las personas podían responder a más de una alternativa

Tabla II CONTACTO INDIRECTO CON PLAGUICIDAS

\begin{tabular}{cc}
\hline Contacto & $\begin{array}{c}\text { Porcentaje } \\
\text { de gestantes }\end{array}$
\end{tabular}

Convive con personas que

trabajan en un lugar donde

se aplican plaguicidas:

$\begin{array}{lc}\text { Sí } & 41,1 \\ \text { No } & 58,9 \\ \mathrm{n}=73 & 100 \\ \text { Almacena plaguicidas: } & \\ \text { Sí } & 21,3 \\ \text { No } & 78,8 \\ \mathrm{n}=80 & 100\end{array}$

* Las personas podían responder a más de una alternativa 
Prevención pre-uso. En la Tabla III, se indican las condiciones de almacenaje de los productos plaguicidas. Si bien nadie señaló guardar los plaguicidas junto a comestibles, menos de una tercera parte de las respuestas indicaron el uso de alguna medida de seguridad en el lugar de almacenamiento cuando este era domiciliario. En cuanto al almacenaje en el lugar de trabajo, en general, las condiciones correctas de almacenamiento se cumplieron. Cabe señalar no obstante, que algunas medidas de seguridad no eran utilizadas en alrededor de un tercio de los lugares de trabajo.
Uso de protección personal durante la aplicación. Doce mujeres reportaron haber aplicado plaguicidas alguna vez y al menos la mitad de ellas no han usado elementos o medidas de protección personal. Los más usados fueron los guantes y el cambiarse de ropa una vez terminado el trabajo. Menos utilizados fueron otros implementos recomendados tales como gafas, mascarillas, traje y zapatos especiales o gorros con visera. Llama la atención, el uso de otras medidas de prevención más domésticas y menos efectivas, como el buzo o camisa de manga larga (Tabla IV).

\section{CONDICIONES DE ALMACENAJE DE PLAGUICIDAS}

\begin{tabular}{|c|c|c|c|c|c|c|}
\hline \multirow{2}{*}{$\begin{array}{l}\text { Condiciones de almacenaje de plaguicidas } \\
\text { En el domicilio: }\end{array}$} & \multicolumn{2}{|c|}{ Sí } & \multicolumn{2}{|c|}{ No } & \multicolumn{2}{|c|}{ Total } \\
\hline & $\mathrm{n}$ & $\%$ & $\mathrm{n}$ & $\%$ & $\mathrm{n}$ & $\%$ \\
\hline Lugar exclusivo para esos fines & 4 & 30,8 & 9 & 69,2 & 13 & 100 \\
\hline Se guardan junto con alimentos o alimentos de animales & 0 & 0 & 12 & 100 & 12 & 100 \\
\hline El lugar tiene piso sólido y lavable & 3 & 23,1 & 10 & 76,9 & 13 & 100 \\
\hline Es un lugar cerrado y ventilado & 4 & 30,8 & 9 & 69,2 & 13 & 100 \\
\hline El lugar tiene extintor & 0 & 0 & 13 & 100 & 13 & 100 \\
\hline Se encuentra señalizado & 1 & 7,7 & 12 & 92,3 & 13 & 100 \\
\hline Es de acceso restringido & 2 & 15,4 & 11 & 84,6 & 13 & 100 \\
\hline En el lugar de trabajo: & $\mathrm{n}$ & $\%$ & $\mathrm{n}$ & $\%$ & $\mathrm{n}$ & $\%$ \\
\hline Lugar exclusivo para esos fines & 11 & 73,3 & 4 & 26,7 & 15 & 100 \\
\hline Se guardan junto con alimentos o alimentos de animales & 0 & 0 & 14 & 100 & 14 & 100 \\
\hline El lugar tiene piso sólido y lavable & 8 & 61,5 & 5 & 38,5 & 13 & 100 \\
\hline Es un lugar cerrado y ventilado & 10 & 71,4 & 4 & 28,6 & 14 & 100 \\
\hline El lugar tiene extintor & 9 & 64,3 & 5 & 35,7 & 14 & 100 \\
\hline Se encuentra señalizado & 10 & 66,7 & 5 & 33,3 & 15 & 100 \\
\hline Es de acceso restringido & 10 & 66,7 & 5 & 33,3 & 15 & 100 \\
\hline
\end{tabular}

Tabla IV

USO DE IMPLEMENTOS DE PROTECCIÓN PERSONAL $(n=12)$

\begin{tabular}{lcc}
\hline Medidas de seguridad & $\begin{array}{c}\text { Si usa } \\
(\%)\end{array}$ & $\begin{array}{c}\text { No usa } \\
(\%)\end{array}$ \\
\hline Guantes & 83,3 & 16,7 \\
Gafas & 50 & 50 \\
Mascarilla & 58,3 & 41,7 \\
Traje especial & 50 & 50 \\
Gorro con visera de legionario & 33,3 & 66,7 \\
Zapatos especiales & 41,7 & 58,3 \\
Buzo pantalón largo & 58,3 & 41,7 \\
Chaqueta o camisa manga larga & 50 & 50 \\
Calcetines o medias & 58,3 & 41,7 \\
Se cambia de ropa cuando termina de aplicar & 66,7 & 33,3 \\
\hline
\end{tabular}


Precauciones post-uso. En cuanto a la percepción de las encuestadas respecto del tiempo para reingresar a una zona fumigada, la mayoría señaló que el período se respeta. No obstante, algo más de una cuarta parte señaló que se respeta tan sólo a veces o nunca. En general $(90,9 \%)$, la ropa utilizada para fumigar, no se lava junto al resto de la ropa de la familia, lo cual es una medida preventiva adecuada. Ninguna mujer señaló reutilizar los envases de plaguicidas vacíos, aunque cerca de la mitad señaló que se queman, tiran a la basura o entierran. Un grupo menor señaló cumplir con la recomendación del triple lavado y envío a centro de acopio (Tabla V).

\section{Tabla V \\ MEDIDAS DE PREVENCIÓN POST APLICACIÓN DE PLAGUICIDAS}

\begin{tabular}{lc}
\hline Espera el tiempo recomendado de reingreso a lugar fumigado: & $\%$ \\
Siempre & 70 \\
A veces & 8 \\
Nunca & 22 \\
$n=50$ & 100 \\
Forma de lavado de ropa: & \\
Junto con otra ropa & 9,1 \\
Aparte de otra ropa, pero en el mismo lavadero & 48,5 \\
Lava en un lugar o lavadora distinta a la doméstica & 42,4 \\
$n=33$ & 100 \\
Forma de eliminación de envases de plaguicidas: & \\
Se reutilizan & 0 \\
Se queman o se tiran a la basura & 54,5 \\
Se lava tres veces y se perforan & 18,2 \\
Se llevan a centro del almacenaje & 27,3 \\
$n=11$ & 100
\end{tabular}

Molestias de salud derivadas del contacto con plaguicidas. A las encuestadas se les consultó si tuvieron molestias de salud derivadas del contacto con plaguicidas. Un $75 \%$ señaló haber sentido al menos una manifestación, siendo la cefalea la más referida, seguida por afecciones gastrointestinales (náuseas y dolor de estómago) y dérmicas. Frente a la pregunta si tienen nociones de primeros auxilios en caso de intoxicación, la mayoría señaló que no. De las gestantes encuestadas que han sentido molestias de salud, algo más de la mitad respondió que descansaron un rato o bebieron líquido y solo una minoría recurrió a un centro de salud (Tabla $\mathrm{VI})$.

Resultado perinatal. Los resultados perinatales se presentan en la Tabla VII. Cerca de la mitad de las gestantes fue sometida a cesárea. El $94,1 \%$ de los recién nacidos fueron de término y el $88,5 \%$ presentó un peso normal al nacer. El 96\% de los recién nacidos presento Apgar entre siete a diez. La mayoría tuvo características normales del cordón umbilical y del estado general. Hubo solo una muerte intrauterina.

\section{DISCUSIÓN}

Un estudio reciente sobre exposición no ocupacional a plaguicidas concluye que la comprensión de las vías de exposición en mujeres que viven en zonas agrícolas es crítico para el estudio de efectos sobre la salud y para el diseño de estrategias de reducción de la exposición (3). En la realidad, las vías de exposición a plaguicidas así como las conductas asociadas a su uso, están escasamente definidas para la mayoría de las comunidades, por lo que estudios de esta naturaleza son esenciales para avanzar el conocimiento necesario para la toma de decisiones y la gestión del riesgo (6). 


\section{Tabla VI \\ SINTOMATOLOGÍA DE SALUD ASOCIADA A CONTACTO CON PLAGUICIDAS, ACCIÓN TOMADA Y NOCIONES DE PRIMEROS AUXILIOS}

Sintomatología inespecífica asociada al contacto con plaguicidas*

Náuseas

Mareos

Vómitos

Visión borrosa

Cefalea

Lesión, picazón a la piel

Sudoración

Calambres piernas, pies

Convulsiones

Salivación excesiva

Cambios estado de ánimo

Debilidad en piernas, pies

Insomnio

Dolor de estómago

Dificultad para respirar

Dolores musculares

$\mathrm{n}=59$

Acción tomada al sentir las molestias*:

Descansar un rato

Tomar leche

Tomar agua o bebida

Asistir a consultorio o buscar atención médica

$\mathrm{n}=27$

Tiene nociones de primeros auxilios frente a intoxicación con plaguicidas:

Sí

No

$\mathrm{n}=70$
Porcentaje que lo mencionó

15,3

6,8

5,1

3,4

30,5

11,9

3,4

3,4

1,7

3,4

6,8

1,7

3,4

10,2

1,7

3,4

Porcentaje que lo mencionó

51,9

7,4

55,6

18,5

Porcentaje que lo mencionó 20

80

100

* Las personas podían responder a más de una alternativa

Un grupo importante de mujeres gestantes en las comunas agrícolas estudiadas declaró haber estado en contacto con plaguicidas alguna vez, mientras que aproximadamente la mitad señaló haber estado expuesta durante el año del estudio o el año anterior, es decir previo o durante su embarazo, demostrando que la exposición a plaguicidas en estas comunas rurales es permanente (Tabla I). Solo una minoría declaró haber aplicado plaguicidas ella misma, lo que coincide con una tarea considerada masculina, o bien podría dejar entrever una posible reticencia a informar el uso de plaguicidas durante la gestación. Destaca también la exposición a plaguicidas a través de la fumigación aérea así como el contacto indirecto a través de vivir con alguien que trabaja con plaguicidas, develando una exposición residencial o indirecta que, muchas veces, se suma a la laboral esporádica o permanente, y frente a la cual hay más bien una resignación a la exposición puesto que no se perciben medidas efectivas de prevención y control.
El contacto con los plaguicidas, tanto laboral como residencial, se da sin las suficientes medidas de prevención (Tablas III, IV y V), observación que se asemeja a lo informado en estudios recientes sobre prácticas de uso de plaguicidas de trabajadores (hombres y mujeres) de otros países, como por ejemplo Australia (7), México (8) o Indonesia (9), destacando también la falta de uso de elementos o equipos de protección personal en gestantes italianas (10).

El número de mujeres que reportaron haber aplicado plaguicidas alguna vez fue bajo, y en este grupo, el uso de elementos de protección personal alcanzó el 50\% aproximadamente. Por norma nacional del Servicio Agrícola y Ganadero, los envases de productos plaguicidas deben contar con etiquetas que indiquen condiciones de uso, riesgos para la salud y medidas de protección. Sin embargo, las etiquetas no son leídas en más de un tercio de las gestantes $(36,4 \%)$, lo cual puede explicar, al menos en parte, la conducta de riesgo. 
Tabla VI

RESULTADO PERINATAL

\begin{tabular}{|c|c|}
\hline Tipo de parto & Porcentaje de g \\
\hline Vaginal & 51,9 \\
\hline Cesárea & 48,1 \\
\hline $\mathrm{n}=54$ & 100 \\
\hline \multicolumn{2}{|c|}{ Edad gestacional } \\
\hline Término & 94,1 \\
\hline Pretérmino & 5,9 \\
\hline$n=51$ & 100 \\
\hline \multicolumn{2}{|c|}{ Muerte intrauterina } \\
\hline Sí & 1,9 \\
\hline No & 98,1 \\
\hline$n=53$ & 100 \\
\hline \multicolumn{2}{|l|}{ Rango de peso } \\
\hline Normal & 88,5 \\
\hline Bajo peso & 0 \\
\hline Macrosómico & 11,5 \\
\hline$n=52$ & 100 \\
\hline \multicolumn{2}{|l|}{ Rango de talla } \\
\hline Pequeño & 7,8 \\
\hline Normal & 90,2 \\
\hline Grande & 2 \\
\hline$n=51$ & 100 \\
\hline \multicolumn{2}{|c|}{ Estado de cordón } \\
\hline Normal & 95 \\
\hline Alterado & 5 \\
\hline$n=20$ & 100 \\
\hline \multicolumn{2}{|c|}{ Estado de salud general del recién nacido } \\
\hline Sano & 88,9 \\
\hline Enfermo & 11,1 \\
\hline$n=54$ & 100 \\
\hline
\end{tabular}

En cuanto a medidas preventivas post-aplicación y que pueden también contribuir al contacto indirecto a plaguicidas, como el lavado de ropa o formas de desechar los envases vacíos, se detectó cerca de un $50 \%$ de conductas que aumentan el contacto, entre ellas, lavar la ropa contaminada en el mismo lavadero o junto con otra ropa. En este punto cabe destacar la aparición de un comentario, que aun cuando no estaba considerado como pregunta de la encuesta, fue mencionado frecuentemente por las entrevistadas y que dice relación con la necesidad de lavar la ropa en forma separada "por mal olor" pero no por protección. Por otro lado, la quema o el desecho de los envases vacíos en la basura general es una práctica también riesgosa y que contradice los esfuerzos de normas de uso de triple lavado y depósito en centros de acopio.

Cerca de un $75 \%$ de las encuestadas señalaron haber sufrido molestias de salud a causa del contacto con plaguicidas, siendo la más recurrente la cefalea, seguido de molestias gástricas y síntomas dermatológicos. Si bien se trata de sintomatología inespecífica, y que de ninguna manera puede asociarse causalmente a la exposición a plaguicidas, este porcentaje refleja la percepción de peligro que tienen las mujeres sobre los plaguicidas. No obstante, esto contrasta con la falta de medidas preventivas. No se encontraron tendencias que destaquen en los antecedentes obstétricos o en el resultado perinatal.

En general, las mujeres que señalan haber tenido estas manifestaciones no acuden al consultorio $\mathrm{u}$ otro servicio asistencial. Aun cuando esto no fue consultado en las entrevistas, en conversaciones anexas, las mujeres consideraron que por un lado, los consultorios tienen baja capacidad resolutiva y por otro, el acudir a un hospital por intoxicación aguda conlleva una notificación obligatoria al MINSAL, 
con la posterior investigación y eventual sanción. En términos sociales, el grupo de gestantes entrevistadas conforman una población relativamente vulnerable, que evita situaciones conflictivas y poco favorables como la notificación obligatoria de intoxicaciones agudas por plaguicidas.

Acorde a observaciones del trabajo de campo, los pequeños agricultores de la zona central de Chile, han sufrido importantes pérdidas económicas a raíz de la sequía de los últimos años. Esta preocupación desplaza la del riesgo de los plaguicidas. Por lo mismo, al haber bajado la producción agrícola por la falta de agua, baja también el uso de agroquímicos y se afecta la actitud preventiva.

Las creencias de las personas son determinantes claves de sus conductas y en especial, la experiencia personal, creencias culturales y nivel de educación pueden ser determinantes de las prácticas de uso de plaguicidas (6). La labor educativa que pueda desarrollarse en los consultorios $u$ otras instituciones vinculadas al agro podría modificar estas creencias para evitar las conductas y prácticas de uso de plaguicidas riesgosas.

\section{CONCLUSIÓN}

En este estudio hemos establecido, por primera vez para Chile, que en comunas rurales puede existir un contacto permanente de la población femenina gestante con plaguicidas no solo laboral, sino que también residencial. Hemos constatado que la exposición a los agrotóxicos sucede sin las medidas de protección adecuadas incrementando el riesgo de exposición a estas sustancias. En Chile, las medidas de vigilancia epidemiológica se focalizan en la intoxicación aguda de la población trabajadora. Es pertinente, entonces desarrollar una labor educativa activa en la que se puedan reforzar conductas y prácticas de uso seguro de plaguicidas, y ampliar el foco poblacional de vigilancia epidemiológica.

Agradecimientos. Nuestros sinceros agradecimientos a las personas, instituciones y organizaciones sociales que dieron su respaldo y/o aportaron de manera sustantiva a la investigación: mujeres gestantes que participaron del estudio, equipos de salud de los CESFAM (en San Pedro: Mónica Quiroga Fernández, Michelly Salles, Julia Lira Pavéz, Aline Cubillo Jaure, Patricia Tapia, Giovanna Arancibia, Anyela Hausheer Aravena y en Chincolco, Pedegua y Hierro Viejo: Elizabeth Rojas, Sara Acuña y equipo paramédico de apoyo) y los Alcaldes de Petorca y Quillota: Sr. Gustavo Valdenegro Rubillo y Dr. Luis Mella Gajardo.

\section{REFERENCIAS}

1. Ministerio de Salud. División de Planificación Sanitaria. Departamento de Epidemiología. Norma Técnica de Vigilancia de Intoxicaciones Agudas por Plaguicidas. REVEP. Santiago. 2007.

2. Silva A, Arancibia M, Arnao M, López P. Percepción de riesgo de plaguicidas en pequeños agricultores y agricultoras de tres comunas de la $\mathrm{V}$ Región Valparaíso. XXIX Acta Científica de Congreso Latinoamericano de Sociología ALAS, Santiago de Chile, 2013, ISBN 978-956-19-0828-4. Disponible en: http://www. actacientifica.servicioit.cl/.

3. Deziel NC, Friesen MC, Hoppin JA, Hines C, Thomas K, Beane Freeman LE. Review of nonoccupational pathways for pesticide exposure in women living in agricultural areas. Environ Health Perspect 2015;123:515-24.

4. Lewis RC, Cantonwine DE, Del Toro LV, Calafat AM, Valentin-Blasini L, Davis MD, Montesano MA, Alshawabkeh AN, Cordero JF, Meeker JD. Distribution and determinants of urinary biomarkers of exposure to organophosphate insecticides in Puerto Rican pregnant women. Sci Total Environ 2015;512-3:337-44.

5. Lorenz AN, Prapamontol T, Narksen W, SrinualN,Barr DB, Riederer AM. Pilot study of pesticide knowledge, attitudes, and practices among pregnant women in northern Thailand. Int $\mathrm{J}$ Environ Res Public Health 2012;9:3365-83.

6. Quandt SA, Doran AM, Rao P,HoppinJane A, SnivelyBM, Thomas $A$. Reporting pesticide assessment results to farm worker families: development, implementation, and evaluation of a risk communication strategy. Environ Health Perspect 2004;112:636-42.

7. MacFarlane A, Chapman A, Benke G, Meaklim J, Sim $M$, McNeil J. Training and other predictors of personal protective equipment use in Australian grain farmers using pesticides. Occup Environ Med 2008;65:141-6.

8. Blanco-Muñoz J, Lacasaña M. Practices in pesticide handling and the use of personal protective equipment in mexican agricultural workers. J Agromed 2011;16:117-26.

9. Yuantari MGC, Van Gestel CAM, Van Straalen NM, Widianarko B, Sunoko HR, Shobib MN. Knowledge, attitude, and practice of Indonesian farmers regarding the use of personal protective equipment against pesticide exposure. Environ Monit Assess 2015;187:142.

10. Giannandrea F, Settimi L, Figa I. The use of personal protective equipment in pregnant greenhouse workers. Occup Med 2008;58:52-7. 